\title{
Hashimoto's Encephalopathy - Clinical Profile and Outcome Study
}

\author{
Lakshmi J. Nair ${ }^{1}$, Ajith S.N. ${ }^{2}$, Ajitha K.C. ${ }^{3}$, Ravikumar Kurup A. ${ }^{4}$ \\ 1,2,3,4 Department of General Medicine, Government Medical College, \\ (Affiliated under Kerala University of Health Sciences), Thiruvananthapuram, Kerala, India.
}

\section{ABSTRACT}

\section{BACKGROUND}

Hashimoto's Encephalopathy (HE) is a rare neuroendocrine syndrome of encephalopathy and high serum antithyroid antibody titre that is responsive to glucocorticoid therapy. Studies are scarce, particularly in the Indian population. This study was intended to find the clinical profile and assess the clinical outcome and response to steroid treatment.

\section{METHODS}

All patients presenting with encephalopathy and positive for antithyroid antibody were included in this study. Their clinical features, thyroid status and laboratory findings were recorded as per protocol. The outcome measures -Mini-Mental State Examination (MMSE) and Modified Ranking Score (MRS) were done at admission and 6th month of follow-up. In addition to descriptive statistics, internal comparisons were made using a t-test or chi-square test. Association between clinical characteristics and outcome variables was measured using logistic regression.

\section{RESULTS}

There were 40 patients ( 11 men and 29 women; mean age, 62 years) diagnosed with Hashimoto's Encephalopathy. Among these patients, 37 (92.5 \%) had cognitive impairment, 27 (67.5\%) had seizures, 12 (30\%) had speech and language dysfunction, 14 (35.0\%) had myoclonus, and 19 (47.5\%) had psychiatric symptoms. The most common thyroid status was euthyroidism (16 patients, $40 \%$ ). $35(87.5 \%)$ patients had elevated CSF protein and 36 (90.0\%) had abnormal EEG findings. On logistic regression, the clinical predictors for the severity of Hashimoto's Encephalopathy were age and CSF elevated protein. Out of 40 patients, $33(82.5 \%)$ patients showed improvement with steroids.

\section{CONCLUSIONS}

HE is an under-diagnosed condition and it should be entertained in any patient with encephalopathy of uncertain aetiology and antithyroid antibodies. A trial of corticosteroids is warranted as it shows a good treatment response to steroids. However, there is no correlation between the neurologic symptoms and signs and the type of antithyroid antibodies or their serum titre. The thyroid status has no bearing on the clinical pattern or the severity of the disease.

\section{KEY WORDS}

Hashimoto Encephalopathy, Autoimmunity, Steroid-Responsive Encephalopathy, Thyroiditis.
Corresponding Author:

Dr. Ajith S. N.,

H. No. B4, Chinmaya, TC-72/2363(1),

Forest Office Lane B Vazhuthacaud,

Thiruvananthapuram-695014,

Kerala, India.

E-mail:drajithsn@gmail.com

DOI: $10.14260 /$ jemds/2022/64

How to Cite This Article:

Nair LJ, Ajith SN, Ajitha KC, et al. Hashimoto's encephalopathy - clinical profile and outcome study. J Evolution Med Dent Sci 2022;11(02):329-334, DOI: $10.14260 / \mathrm{jemds} / 2022 / 64$

Submission 18-01-2022,

Peer Review 23-01-2022,

Acceptance 01-02-2022,

Published 03-02-2022.

Copyright (c) 2022 Lakshmi J. Nair et al. This is an open access article distributed under Creative Commons Attribution License [Attribution 4.0 International (CC BY 4.0)] 


\section{BACKGROUND}

Hashimoto's Encephalopathy (HE) was first described in 1966 by Lord Brain, since then it has gained prominence in the differential diagnosis of encephalopathy of unknown origin. ${ }^{1}$ Hashimoto's Encephalopathy is a rare neuropsychiatric syndrome, more common in women, associated with serologic evidence of anti-thyroid antibodies when other causes of encephalopathy are excluded. It may have an acute onset, characterized by episodes of cerebral ischemia, seizure, and psychosis, or it may present as an indolent form with depression, cognitive decline, myoclonus, tremors, and fluctuations in the level of consciousness. The term has also been used interchangeably with other terms such as Hashimoto's Encephalitis, ${ }^{2}$ Steroid responsive encephalopathy associated with autoimmune thyroiditis (SREAT) $^{3}$ and Nonvasculitic autoimmune inflammatory meningoencephalitis (NAIM). ${ }^{4}$ The lack of clinical diagnostic criteria, uniformity in the clinical presentation, and most importantly a solid understanding of etiological and pathophysiological mechanisms have resulted in a dazzling array of case reports entitled "Hashimoto's Encephalopathy," the consequences being an ever-expanding definition of the term and application to any encephalopathy or psychiatric condition associated with antithyroid antibodies.

The incidence and prevalence of Hashimoto's encephalopathy are not known but one study of unexplained encephalopathies found a prevalence of 2.1/100,000 in the general population (Ferracci et al., 2004). ${ }^{5}$ There are no reports of familial Hashimoto's Encephalopathy. Once infectious causes are excluded, an autoimmune process may be suspected based on inflammatory and autoimmune markers in the serum / CSF and meningeal / parenchymal abnormalities on MRI of the brain. Hashimoto's Encephalopathy is a part of the large spectrum of autoimmune encephalopathies, which should be suspected when there is a high serum level of thyroperoxidase (TPO) antibody in a patient with a matching clinical setting and is highly responsive to steroids.

Although high serum levels of thyroperoxidase (TPO) antibody, formerly known as an anti-microsomal antibody, are often found in patients with SREAT, this organ-specific autoantibody is a serologic marker for the more common condition of autoimmune (Hashimoto) thyroiditis and a common marker of autoimmunity in most autoimmune neurologic disorders. ${ }^{6}$

Therefore a direct causal relationship between thyroid antibodies and encephalopathy is unlikely. Numerous case reports, small series, and meta-analysis have characterized this entity further.

The literature thus far has not provided sufficient data on issues to aid in the diagnosis of this disorder and guide its management.7,8,9 We sought to address some of these issues by analysing data on a series of patients in whom the diagnosis of Hashimoto's encephalopathy was made at Government Medical College, Trivandrum from 2020 to 2021. Our purpose was to characterize the specific clinical manifestations that could point towards its diagnosis. We also focused on the thyroid function of these patients and included various presenting features of encephalopathy particularly involuntary movements in the study. This study hopes to change the approach to non-infective encephalopathy, aiding in diagnosing more cases of Hashimoto's encephalopathy and ascertaining the clinical and serological factors responsible for the severity and outcome of the disease. The study also has prospectively looked into the clinical response to treatment with steroids and thyroxine after six months.

\section{Objectives}

1. To characterize the clinical profile, radiological and electrophysiological correlation in patients with Hashimoto's encephalopathy under the Department of Internal Medicine, Government Medical College, Thiruvananthapuram.

2. To examine the correlation between Serum Thyroid Peroxidase titres and Thyroid hormone levels with clinical exacerbation.

3. To ascertain the outcome of Hashimoto's encephalopathy and evaluate its progression to chronic neurodegenerative disease.

\section{METHODS}

This is a descriptive observational study on the clinical profile and outcome of Hashimoto's encephalopathy with follow up for six months. A hospital-based study was carried in the general medical wards and medical ICU, Medical College, Trivandrum for a period of one year, from March 2020 to April 2021.

\section{Sample Size and Sampling Technique}

All patients meeting the eligibility criteria, and who reported at the study setting were enrolled in the study (census type) and the effective sample size came to be 40 .

\section{Inclusion Criteria}

All patients required fulfilment of the following criteria:

a. Encephalopathy manifested by cognitive impairment and 1 or more of the following: neuropsychiatric features (eg. Hallucinations, delusions, or paranoia), myoclonus, generalized tonic-clonic or partial seizures, or focal neurologic deficits;

b. Presence of serum thyroid antibody (TPO or microsomal);

c. Euthyroid status [serum thyroid-stimulating hormone (TSH), $\quad 0.25-5.0 \mathrm{mlU} / \mathrm{L}]$ or hyperthyroidism or hypothyroidism that would not account for the encephalopathy.

d. The absence of structural, infectious or other metabolic factors could explain the altered mental status and its response to steroids.

\section{Exclusion Criteria}

a. Patients who were not willing to give consent;

b. No evidence in blood, urine, or CSF analyses of an infectious, toxic, metabolic, or neoplastic process;

c. No relevant serologic evidence of other autoimmune encephalopathy or other currently recognized 
paraneoplastic autoantibodies to indicate another diagnosis; and

d. No findings on neuroimaging studies indicating vascular, neoplastic, or other structural lesions to explain the encephalopathy;

e. Patients with other known neurological diseases like stroke and dementia.

\section{Statistical Analysis}

The data was collected and analyzed using statistical tools. Mean median, percentage sampling were used for quantitative analysis and Statistical Package for Social Sciences, SPSS (version 23) was used for qualitative analysis to derive the values of probability ( $\mathrm{p}$-value). $\mathrm{P}<0.05$ was considered statistically significant. The chi-square test was used appropriately for the analysis of variables.

\section{RESULTS}

The study was conducted on 40 willing patients admitted to medical wards diagnosed with Hashimoto's encephalopathy. The data were collected based on clinical examination and laboratory investigations during the study period. The tools employed in the data collection included Mini-Mental State Examination (MMSE), modified Rankin scale (MRS) and imaging techniques. Basic and clinical characteristics including demographic data such as age, sex, concurrent illness, clinical symptomatology were collected and categorized. The serum anti-thyroid peroxidase levels and other laboratory investigations were obtained within 48 hours of admission. Standard methods were adopted in the estimation of serum T3, T4, TSH and TPO levels. Neurological impairment and the severity of the disease were assessed using the MMSE and Rankin scale.

\section{Background Characteristics}

1. Age

Out of the 40 patients studied, the minimum age was 31 and the highest age was 81 years. The mean age was $62.4 \pm 8.62$. Eighty-five percent of the patients were of the age between 50 - 70 years. Only $5 \%$ was below the age of 50 , indicating that the prevalence increases with age.

\section{Gender}

Of the 40 patients studied. $72.5 \%$ were females and $27.5 \%$ were males. The male to female ratio was $1: 3$

\section{Laboratory Characteristics of Hashimoto's Encephalopathy}

\begin{tabular}{|ccc|}
\hline Investigations & Frequency & Percentage \\
Elevated ESR & 5 & 11.1 \\
Elevated CRP & 4 & 8.8 \\
Elevated ANA/RA & 2 & 4.4 \\
Elevated Liver enzymes & 13 & 28.9 \\
Antithyroglobulin Antibody & 31 & 68.9 \\
\hline \multicolumn{2}{|}{ Table 1. General Features of Patients with Hashimoto's } \\
& Encephalopathy \\
\hline
\end{tabular}

4. Hyponatremia in Hashimoto's Encephalopathy Out of the 40 patients studied, $65 \%$ had Hyponatremia.

\section{Thyroid status}

The thyroid status of the patients were as follows: 16 patients (40\%) were euthyroid, 19 (47.5\%) hypothyroid, and 5 (12.5 $\%$ ) were hyperthyroid

\section{Spectrum of Neurological Manifestations of $H E$}

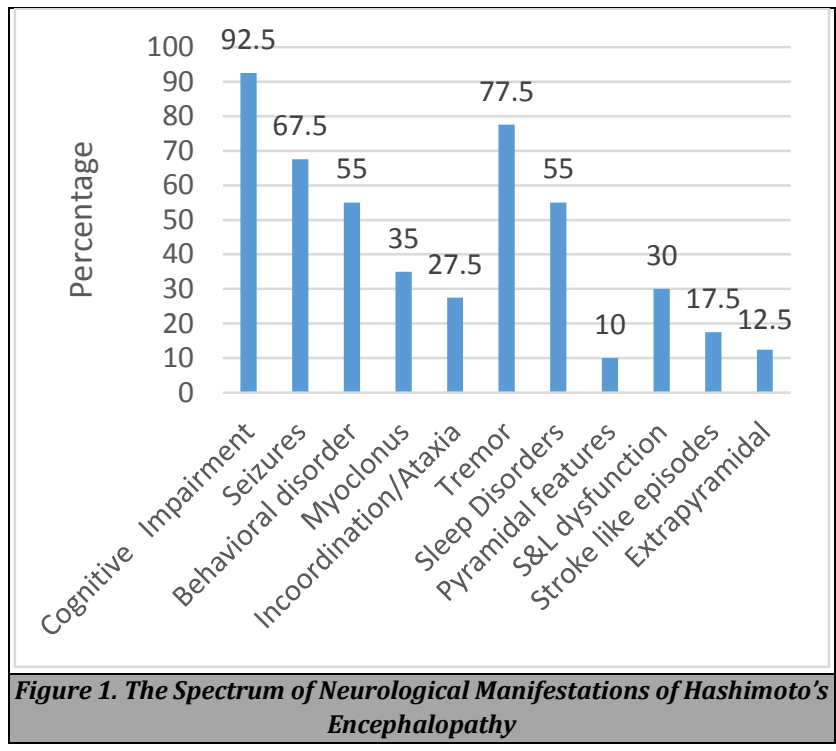

7. Neuropsychiatric Manifestations

\begin{tabular}{|ccccccc|}
\hline Neuropsychiatric Manifestation & \multicolumn{2}{l}{ Yes } & \multicolumn{2}{l}{ No } & \multicolumn{2}{c|}{ Total } \\
& N & $\%$ & N & $\%$ & N & $\%$ \\
Depression & 8 & $20 \%$ & 32 & $80 \%$ & 40 & 100.0 \\
Anxiety & 2 & $5 \%$ & 38 & $95 \%$ & 40 & 100.0 \\
Psychosis & 4 & $10 \%$ & 36 & $90 \%$ & 40 & 100.0 \\
Apathy & 5 & $12.5 \%$ & 35 & $87.5 \%$ & 40 & 100.0 \\
\hline Table 2. Percentage Distribution According to the & \\
Neuropsychiatric Manifestations & & \\
\hline
\end{tabular}

8. Serum Anti Thyroid Peroxidase Levels

All 40 patients $(100 \%)$ had positive serum Anti Thyroid Peroxidase test (TPO). Out of these patients, 22 of them (55 $\%$ ) had high titers of serum Anti TPO levels (> $600 \mathrm{IU} / \mathrm{ml})$ and 31 patients (77.5\%) had elevated CSF Anti TPO.

\section{Cerebrospinal Fluid Study}

\begin{tabular}{|ccc|}
\hline Cerebrospinal Fluid & Frequency & Percent \\
\hline Elevated protein & 35 & $87.5 \%$ \\
Elevated WBC count ( $>4$ cells/ml) & 2 & $5 \%$ \\
Normal study & 6 & $15 \%$ \\
Albuminocytological dissociation & 34 & $85 \%$ \\
CSF Anti TP0+ & 31 & $77.5 \%$ \\
\hline Table 3. Percentage Distribution of the CSF Analysis \\
of the Study Sample \\
\hline
\end{tabular}

\section{Electroencephalography (EEG) Findings}

\begin{tabular}{|ccc|}
\hline EEG Findings & Frequency & $\%$ \\
Normal & 4 & $10 \%$ \\
Generalised Slowing & 22 & $55 \%$ \\
Focal intermittent slow waves & 4 & $10 \%$ \\
Epileptiform discharges & 5 & $12.5 \%$ \\
\hline Triphasic waves & 1 & $2.5 \%$ \\
\hline Table 4. Percentage distribution of the sample according to \\
& the EEG findings \\
\hline
\end{tabular}




\section{Mini-Mental State Examination}

All the 40 patients underwent a detailed mini-mental state examination at the time of admission and at follow up after 6 months. The following were the results:

\begin{tabular}{|ccc|}
\hline MMSE & At Admission & At 6 Month Follow up \\
Number of values & 40 & 40 \\
Minimum & 5 & 10 \\
Maximum & 22 & 26 \\
Range & 17 & 16 \\
Mean & 15.05 & 18.2 \\
Std. Deviation & 4.181 & 4.09 \\
Median & 15 & 18 \\
Wilcoxon Signed Rank Test & $\mathrm{P}=<0.001$ \\
\hline Table 5. MMSE Score Details of the Sample at the Time of \\
Admission and at Follow up after 6 Months.
\end{tabular}

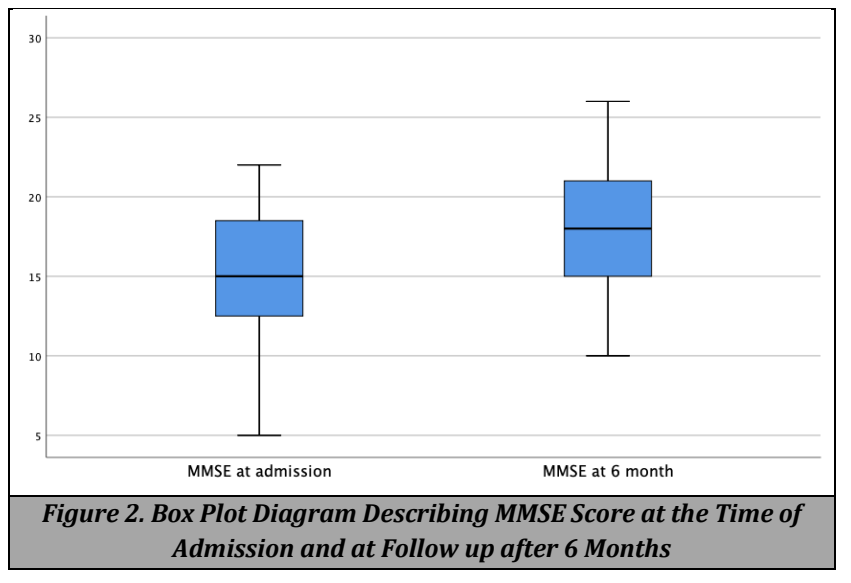

The lower and upper end of the whisker shows the minimum and maximum MMSE at each time interval. The lower border of the box represents the first quartile and the upper border of the box represents the third quartile of MMSE and the middle line, the line of separation of the two coloured boxes, represents the median MMSE score.

\section{Type of Treatment Given}

Out of 40 Patients, $50 \%$ that is 20 patients received steroid plus thyroxine and the other $50 \%$ received thyroxine alone.

\section{MMSE Score and Type of Treatment Given}

Out of 40 patients, 33 patients showed clinical improvement (change of MMSE $>1$ ) after steroid treatment. Thus around $82.5 \%$ of the patients showed improvement with steroids. However, two patients (5\%) worsened after treatment with steroids at the $6^{\text {th }}$ month follow up.

\section{Association between Severities of Hashimoto's} Encephalopathy and Various Clinical Parameters

\begin{tabular}{|c|c|c|c|c|c|c|c|c|c|}
\hline \multirow{3}{*}{ Presentation } & \multicolumn{4}{|c|}{ MMSE at Admission } & \multirow{2}{*}{\multicolumn{2}{|c|}{ Total }} & \multirow{3}{*}{$\chi^{2}$} & \multirow{3}{*}{ df } & \multirow{3}{*}{$\mathbf{P}$} \\
\hline & \multicolumn{2}{|c|}{ No/Mild } & \multicolumn{2}{|c|}{ Severe } & & & & & \\
\hline & $\mathbf{N}$ & $\%$ & $\mathbf{N}$ & $\%$ & $\mathbf{N}$ & $\%$ & & & \\
\hline Age $>60$ & 6 & 46.2 & 21 & 77.8 & 27 & 67.5 & 4.00 & 1 & 0.045 \\
\hline Female & 10 & 76.9 & 19 & 70.4 & 29 & 72.5 & 0.19 & 1 & 0.664 \\
\hline Overt Hypothyroid & 2 & 15.4 & 4 & 14.8 & 6 & 15 & 1.07 & 4 & 0.899 \\
\hline S. TPO High titre & 7 & 53.8 & 15 & 55.6 & 22 & 55 & 0.01 & 1 & 0.919 \\
\hline CSF elevated protein Present & 8 & 61.5 & 27 & 100 & 35 & 87.5 & 11.9 & 1 & 0.001 \\
\hline Acute presentation & 8 & 61.5 & 15 & 55.6 & 23 & 57.5 & 0.13 & 1 & 0.720 \\
\hline Psychiatric Manifestations & 3 & 23.1 & 3 & 11.1 & 6 & 15 & 0.99 & 1 & 0.321 \\
\hline Hyponatremia & 9 & 69.2 & 17 & 63 & 26 & 65 & 0.15 & 1 & 0.697 \\
\hline 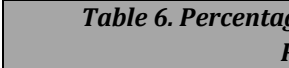 & . & 20 & ent & $x^{2}+2$ & & & & & \\
\hline
\end{tabular}

\section{DISCUSSION}

The term Hashimoto's encephalopathy was used to describe a syndrome of persistent or fluctuating neurological and particularly neuropsychological deficits in Hashimoto's thyroiditis, despite the thyroid hormone levels being within the normal range. Since its first description in 1966, several cases of Hashimoto's encephalopathy have been reported. But this entity continues to be underdiagnosed. Hashimoto's encephalopathy has huge clinical relevance since it is not uncommon and shows a very gratifying response to immunosuppressive treatment. Studies conducted in this field shows that all patients were assigned an alternative misdiagnosis at presentation, most commonly viral encephalitis, Creutzfeldt-Jakob disease or degenerative dementia. ${ }^{10}$

In the present study, $40 \%$ of the patients with Hashimoto's encephalopathy were clinically and biochemically euthyroid. $32.5 \%$ had subclinical hypothyroidism and $15 \%$ had overt hypothyroidism. And around $2.5 \%$ were hyperthyroid. Unlike what its name might suggest, several cases of HE have been described in patients with Graves' disease. In our review of the literature, $10 \%$ of patients had hyperthyroidism, which reinforces the fact that the disease is not associated exclusively with Hashimoto's thyroiditis, but also with other autoimmune thyroid diseases. $^{11}$ For these reasons, it is more appropriate to use the term "steroid-responsive encephalopathy associated with autoimmune thyroiditis (SREAT), ${ }^{3}$ rather than Hashimoto's encephalopathy.

Thyroid status does not appear to influence the mechanism of the disease since our study shows that most patients presented with normal thyroid function on diagnosis, as well as levels unrelated to the course of the disease. Literature states that though Hashimoto's encephalopathy may be associated with hypothyroidism, thyroid function is usually clinically and biochemically normal. ${ }^{12}$ Neurologic symptoms and signs are similar in patients irrespective of their thyroid status. ${ }^{13}$ Our study also confirms that thyroid status has no bearing on the clinical pattern or the severity of the disease.

The present study showed that the most common presentation of Hashimoto's encephalopathy was cognitive impairment (92.5\%), followed by tremor (77.5\%) and myoclonus was found in $35 \%$ of the patients. Seizures appeared to be extremely common in Hashimoto's encephalopathy and deserved consideration in the differential diagnosis of adult-onset recurrent epileptic seizures.

The majority of the patients (65\%) in the present study had hyponatremia. They were further evaluated with thyroid function tests to see if there was any association between hypothyroidism and hyponatremia. Literature gives a strong correlation between hypothyroidism and hyponatremia. Hyponatremia is reported in up to $10 \%$ of hypothyroid patients, although it is usually mild and rarely causes symptoms. The relationship between hypothyroidism and hyponatremia was recently questioned by Warner et al. ${ }^{14}$ Although they showed a statistical association between hyponatremia and hypothyroidism (for every $10 \mathrm{mU} / \mathrm{l}$ rise in thyroid-stimulating hormone, serum sodium is decreased by 
$0.14 \mathrm{mmol} / 1$ ), the clinical relevance of this association seems minute. If the relationship does exist, low cardiac output and/or a low glomerular filtration rate could explain its pathophysiology.

All previous studies had common TPO positivity in almost all patients with Hashimoto's encephalopathy. However, antiTPO antibodies have also been found in rheumatoid arthritis, insulin-dependent diabetes mellitus, and a low percentage of euthyroid subjects ( $14.4 \%$ in men and $25.8 \%$ in women).

Our study concludes that there was no significant relationship between the type of antibody present and the neurological findings. Some studies suggested that antithyroid antibody level elevation is proportional to the degree of disease activity since levels have been shown to decrease after treatment with steroids. ${ }^{15}$ However, other studies showed that antibody levels increased again after withdrawal of steroids without a clinical relapse and therefore argued against this relationship. ${ }^{3}$ Moreover, there is no evidence that the antibodies play a specific role in the pathogenesis of HE, but rather possibly represent a marker for another autoimmune process involving the brain. Recent studies also demonstrated the presence of antithyroid antibodies in the CSF of subjects with Hashimoto's encephalopathy. Intrathecal synthesis of the antibodies was suggested, and a lack of association between antibody titers and disease severity and stage of treatment was reported. ${ }^{16}$

Cerebral and thyroid dysfunction may have a common immune dysregulation, e.g. antibodies sharing a common $\mathrm{T}$ cell epitope. On the other hand, neuronal dysfunction may be a consequence of thyroid disease. The mechanism is, however unclear. No findings are supporting a role for thyroid autoantibodies. The most relevant antibodies against thyroid peroxidase do not seem to be the mediators of cytotoxicity to the thyroid gland.

Of the 36 patients who had EEG data, 36 presented with some type of changes, the most frequent of which was a generalized slowing of waves, present in 22 (55\%). Other findings included frontal intermittent rhythmic delta activity (FIRDA) and/or delta and theta activity, focal and intermittent slow waves (4 patients: $10 \%$ ), epileptiform discharges, and triphasic waves. The EEG adds little to the diagnosis of HE, as none of the findings is specific. The EEG helps evaluate and follow patients with HE in reflecting the degree of CNS involvement, in determining whether their condition is better or worse, and in ruling out other causes.

Regarding the mini-mental status examination (MMSE) our study showed that the mean MMSE at the time of admission was 15.05 with a standard deviation of 4.181. The minimum and maximum MMSE scores were 5 and 22 respectively. The mean MMSE at follow up of 6 months was 18.2 , the difference being statistically significant $(\mathrm{P}<0.001)$. This is consistent with the generally accepted view that treatment with steroids promotes marked clinical improvement and usually infers a good prognosis.

Out of the 40 patients in our study, $50 \%$ (20) of them were treated with both Inj. methylprednisolone and thyroxine. The remaining $50 \%$ (20) were treated with intravenous methylprednisolone alone. Our study shows that there was no difference in the response to treatment with respect to the treatment option.

A review of the literature reported improvement in $98 \%$ of cases treated with steroids, $92 \%$ treated with steroids and levothyroxine, and $67 \%$ treated with levothyroxine, while 9 $\%$ of cases did not improve with any of the above combinations. $^{8} 90 \%$ of cases stayed in remission even after discontinuation of treatment. Cases of the relapsing/remitting type usually did better than the progressive type, a few of which developed irreversible persistent cognitive impairment. ${ }^{17}$

In view of this study, it may be suggested that the diagnosis of HE should be based on neuropsychiatric manifestations associated with the following findings, provided that other causes of encephalopathy are excluded: 1) presence of high titres of antithyroid antibodies in the serum or CSF; 2) no change in CSF indicative of an infectious, vascular, or neoplastic aetiology; 3) nonspecific MRI or CT scans, and 4) good response to immunosuppressive therapy. We have observed that, in general, HE responds promptly to treatment and appears to be a disease with a good prognosis. There was complete remission of symptoms in most patients, followed by normalization of neuroimaging and EEG after corticosteroid therapy. However, there may be a form of partial remission, with the persistence of the neurological and /or imaging changes, and even death from complications of the disease. Treatment should be based on the use of corticosteroids, most commonly, IV methylprednisolone 0.5 $1 \mathrm{~g} /$ day for 3 to 5 days, with oral prednisone at a high initial dose (1-2 mg/kg/day), followed by gradual reduction, based on clinical improvement. ${ }^{18}$

\section{CONCLUSIONS}

Hashimoto's encephalopathy is an underdiagnosed condition. The common clinical manifestations observed from this study in the decreasing order of frequency are cognitive impairment, tremors, seizures, behavioural disorder, speech, language dysfunction and myoclonus.

A diagnosis of Hashimoto's encephalopathy should be entertained in any patient with encephalopathy of uncertain aetiology and positive antithyroid antibodies.

Hashimoto's encephalopathy is characterised by elevated CSF protein and abnormal EEG and it is usually associated with the euthyroid state but may be associated with hypothyroidism also.

The thyroid status has no bearing on the clinical pattern or the severity of the disease. There is no correlation between the neurologic symptoms and signs and the type of antithyroid antibodies or its serum titre. A trial of corticosteroids is warranted as this study showed a good treatment response to steroids.

\section{REFERENCES}

[1] Brain L, Jellinek E, Ball K. Hashimoto's disease and encephalopathy. Lancet 1966;2(7462):512-4.

[2] Seipelt M, Zerr I, Nau R, et al. Hashimoto's encephalitis as a differential diagnosis of Creutzfeldt-Jakob disease. J Neurol Neurosurg Psychiatry 1999;66(2):172-6.

[3] Castillo P, Woodruff B, Caselli R, et al. Steroid-responsive encephalopathy associated with autoimmune thyroiditis. Arch Neurol 2006;63(2):197-202. 
[4] Caselli RJ, Boeve BF, Scheithauer BW, et al. Nonvasculitic autoimmune inflammatory meningoencephalitis (NAIM): a reversible form of encephalopathy. Neurology 1999;53(7):1579-81.

[5] Ferracci F, Moretto G, Candeago R, et al. Antithyroid antibodies in the CSF: their role in the pathogenesis of Hashimoto's encephalopathy. Neurology 2003;60(4):712-4.

[6] Marcocci C, Chiovato L. Thyroid-directed antibodies. In: Braberman L, ed. The Thyroid. Vol 8. Baltimore, Md: Williams \& Wilkins 2000:414-31.

[7] Schiess N, Pardo CA. Hashimoto's encephalopathy. Ann N Y Acad Sci 2008;1142:254-65.

[8] Sunil G, Mariash C. Hashimoto's encephalitis. The Journal of Clinical Endocrinology Metabolism 2001;86(2):947-7.

[9] Chong JY, Rowland LP, Utiger RD. Hashimoto encephalopathy: syndrome or myth? Arch Neurol 2003;60(2):164-71.

[10] Ishii K, Hayashi A, Tamaoka A, et al. Case report: thyrotropin-releasing hormone-induced myoclonus and tremor in a patient with Hashimoto's encephalopathy. Am J Med Sci 1995;310(5):202-5.

[11] Boeve BF, Castillo PR, Caselli JR, et al. Steroid-responsive encephalopathy associated with thyroid autoimmunity: outcome with immunomodulatory therapy. J Neurol Sciences 2001;187:S441.
[12] Tang Y, Xing Y, Zhang J, et al. Clinical features and outcomes of patients with Hashimoto's encephalopathy. Zhonghua Yi Xue Za Zhi 2014;94(9):670-3.

[13] Fukunaga M, Murai H, Minohara M, et al. Hashimoto's encephalopathy with bilateral pallidal lesions presenting memory disturbance and executive dysfunction. Rinsho Shinkeigaku 2006;46(8):568-71.

[14] Warner M, Holding S, Kilpatrick E. The effect of newly diagnosed hypothyroidism on serum sodium concentrations: a retrospective study. Clin Endocrinol 2006;64(5):598-9.

[15] Canton A, de Fabregas 0 , Tintore $M$, et al. Encephalopathy associated to autoimmune thyroid disease: a more appropriate term for an underestimated condition? J Neurol Sci 2000;176(1):65-9.

[16] Ferracci F, Bertiato G, Moretto G. Hashimoto's encephalopathy: epidemiologic data and pathogenetic considerations. J Neurol Sci 2004;217(2):165-8.

[17] Kothbauer-Margreiter I, Sturzenegger M, Komor J, et al. Encephalopathy associated with Hashimot thyroiditis: diagnosis and treatment. J Neurol 1996;243(8):585-93.

[18] Chang T, Riffsy MTM, Gunaratne PS. Hashimoto encephalopathy: clinical and MRI improvement following high-dose corticosteroid therapy. Neurologist 2010;16(6):394-6. 